\title{
Maximizing a class of submodular utility functions
}

\author{
Shabbir Ahmed • Alper Atamtürk
}

Received: 4 April 2008 / Accepted: 3 July 2009 / Published online: 8 August 2009

(C) The Author(s) 2009. This article is published with open access at Springerlink.com

\begin{abstract}
Given a finite ground set $N$ and a value vector $a \in \mathbb{R}^{N}$, we consider optimization problems involving maximization of a submodular set utility function of the form $h(S)=f\left(\sum_{i \in S} a_{i}\right), S \subseteq N$, where $f$ is a strictly concave, increasing, differentiable function. This utility function appears frequently in combinatorial optimization problems when modeling risk aversion and decreasing marginal preferences, for instance, in risk-averse capital budgeting under uncertainty, competitive facility location, and combinatorial auctions. These problems can be formulated as linear mixed 0-1 programs. However, the standard formulation of these problems using submodular inequalities is ineffective for their solution, except for very small instances. In this paper, we perform a polyhedral analysis of a relevant mixed-integer set and, by exploiting the structure of the utility function $h$, strengthen the standard submodular formulation significantly. We show the lifting problem of the submodular inequalities to be a submodular maximization problem with a special structure solvable by a greedy algorithm, which leads to an easily-computable strengthening by subadditive lifting
\end{abstract}

The research of the first author has been supported, in part, by Grant \# FA9550-08-1-0117 from the Air Force Office of Scientific Research. The research of the second author has been supported, in part, by Grant \# DMI0700203 from the National Science Foundation. The second author is grateful for the hospitality of the Georgia Institute of Technology, where part of this research was conducted.

S. Ahmed

School of Industrial and Systems Engineering,

Georgia Institute of Technology, Atlanta, GA 30332, USA

e-mail: sahmed@isye.gatech.edu

A. Atamtürk $(\varangle)$

Department of Industrial Engineering and Operations Research,

University of California, Berkeley, CA 94720-1777, USA

e-mail: atamturk@berkeley.edu 
of the inequalities. Computational experiments on expected utility maximization in capital budgeting show the effectiveness of the new formulation.

Mathematics Subject Classification (2000) $\quad 90 \mathrm{C} 57 \cdot 91 \mathrm{~B} 16 \cdot 91 \mathrm{~B} 28$

Keywords Expected utility maximization - Combinatorial auctions - Competitive facility location $\cdot$ Submodular function maximization $\cdot$ Polyhedra

\section{Introduction}

Given a finite ground set $N$ and a value vector $a \in \mathbb{R}^{N}$, consider a set utility function $h: 2^{N} \rightarrow \mathbb{R}$ of the form

$$
h(S)=f\left(\sum_{i \in S} a_{i}\right), \quad S \subseteq N,
$$

where $f: \mathbb{R} \rightarrow \mathbb{R}$ is a strictly concave, increasing, and differentiable function. Such utility functions arise frequently in modeling risk aversion and decreasing marginal preferences in combinatorial optimization problems. In the following, we present a few examples on expected utility maximization, competitive facility location, and combinatorial auctions with submodular utilities.

\subsection{Expected utility with discrete choices}

In expected utility theory $[23,24]$ utility functions are used for representing an investor's risk preferences against uncertain outcomes. Concave utility functions imply risk-averse preferences as utility of the (certain) expectation of the outcomes is higher than the expectation of the utilities of the uncertain outcomes. While most research in this area is concerned with a convex decision set, our results are applicable for expected utility maximization when decisions are discrete, such as investments in infrastructure projects, venture capital, and private equity deals. Consider a set $N$ of investment possibilities with uncertain future values. Let $v_{i} \in \mathbb{R}^{N}$ be the value vector for the investments under scenario $i$ with probability $\pi_{i}, i=1, \ldots, m$. Then the expected utility maximization problem of a risk-averse investor can be stated as

$$
\max \left\{\sum_{i=1}^{m} \pi_{i} f\left(v_{i} x\right): x \in X \subseteq\{0,1\}^{N}\right\},
$$

where $f$ is a concave, increasing utility function and $X$ denotes the set of feasible investments. Klastorin [14], Mehrez and Sinuany-Stern [19], Weingartner [26] consider various versions of this problem. A convenient and commonly used [5] utility function for risk aversion is the (negative) exponential utility function: $f(z)=$ $1-\exp (-z / \lambda)$, where $\lambda>0$ is the risk tolerance parameter, with larger $\lambda$ 's representing lesser risk aversion. 


\subsection{Competitive facility location}

Competitive facility location models aim to maximize the total demand captured by the facilities of a company, when facilities compete with each other for demand. While opening a new facility expands the market of the company, it also has the effect of "cannibalization" by reducing demand for other facilities. Consider the problem of choosing $k$ facility locations from a set $N$ of potential sites to serve a set $M$ of markets with maximum demand $d_{i}, i \in M$. In marketing and location literature, a common way of measuring the demand captured by the facilities uses a Huff-type [13] utility $u_{i j}$, for $i \in M, j \in N$, which is a non-decreasing function of attractiveness and distance of the facilities to the customers. Letting $x \in\{0,1\}^{N}$ denote an indicator vector for opened facilities, the proportion of demand in market $i$ captured by the opened set of facilities is given by $f_{i}\left(\sum_{j \in N} u_{i j} x_{j}\right)$, where $f_{i}$ is a strictly concave and increasing function to capture the effect of decreasing marginal utility of additional facilities $[1,4]$. Then the competitive facility location problem is formulated as

$$
\max \left\{\sum_{i \in M} f_{i}\left(\sum_{j \in N} u_{i j} x_{j}\right) d_{i}: \sum_{j \in N} x_{j}=k, x \in\{0,1\}^{N}\right\} .
$$

\subsection{Combinatorial auctions and welfare with submodular utilities}

In combinatorial auctions bidders with nonlinear utilities on subsets of items are allowed to bid for subsets of the items. A common problem of interest is distributing the set of items $(N)$ to the set of bidders $(M)$ so as to maximize the social welfare, measured as the sum of utilities of the bidders. Letting $x_{i j}$ denote whether item $j$ is allocated to bidder $i$, this problem can be formulated as

$$
\max \left\{\sum_{i \in M} f_{i}\left(\sum_{j \in N} a_{i j} x_{i j}\right): x \in X \subseteq\{0,1\}^{M N}\right\},
$$

where $a_{i j}$ denotes the value of item $j$ to bidder $i$ and $f_{i}$ is an increasing and concave function implying decreasing marginal preference of an additional allocation to a bidder-hence submodular utilities. Fiege [7], Lehman et al. [16], Vondrak [25] give approximation algorithms for maximizing social welfare with submodular utilities.

If the utilities of the bidders are unknown to the auctioneer, one may hold an iterative auction, in which given the prices $p \in \mathbb{R}^{N}$ for the items, each player bids for combinations of items that maximize the difference between their utility and prices by responding to so-called demand queries, i.e., by solving the optimization problem

$$
\max \left\{f_{i}(a x)-p x: x \in X^{i} \subseteq\{0,1\}^{N}\right\}, i \in M,
$$

where $X^{i}$ denotes the constraints of bidder $i$. This approach leads to a set packing formulation of the welfare maximization problem, populated with new bids as the prices 
change during the iterations of the auction. Dobzinski et al. [6], Lehman et al. [17] give approximation algorithms for welfare maximization with demand queries using submodular utility functions.

Typically the set function $h$ is submodular. Hence, the above-stated problems involve the maximization of a submodular function, which is known to be $\mathcal{N} \mathcal{P}$-hard as the max-cut problem is a special case. One line of research addressing submodular function maximization is to develop approximation algorithms [8,20,22]. The interest of this paper, however, is mathematical programming formulations amenable for an exact solution with branch-and-bound methods. For submodular maximization Nemhauser and Wolsey [21, p. 710] give a linear mixed 0-1 formulation with exponentially many inequalities. This general formulation has been applied to a number of different submodular maximization problems, including the quadratic partitioning problem [15] and the fixed-charge network flow problem [29].

Even though the utility function $h$ is not necessarily submodular for arbitrary $a \in \mathbb{R}^{N}$, by complementing binary variables, a suitable submodular function can instead be used in order to utilize the submodular formulation of Nemhauser and Wolsey (see Sect. 3). However, this general submodular formulation has a weak linear programming bound and is ineffective for solving all but very small instances. In this paper, by exploiting the special structure of $h$, we significantly strengthen the general submodular inequality formulation for maximization of this class of utility functions.

In particular, we consider the relevant mixed-integer set

$$
F:=\left\{x \in\{0,1\}^{N}, w \in \mathbb{R}: w \leq f(a x+d)\right\}
$$

where $a \in \mathbb{R}^{N}$ and $d \in \mathbb{R}$. It is useful to include the constant term $d$ for generality, which will become necessary for studying the associated lifting problems with parametric right-hand-side. Because $F$ is the union of a finite set of polyhedra (one for each value of the binary vector $x$ ) with a common recession cone, the convex hull of $F$ is a polyhedral set. Thus $F$ can be expressed using linear inequalities. While differentiability assumption on $f$ can be relaxed, for a simpler characterization of solutions to the continuous relaxation, it is convenient to assume so. In the remainder of this paper we develop linear valid inequalities for $F$ that are stronger than the general submodular inequalities of Nemhauser and Wolsey [21].

In Sect. 2 we show that optimizing a linear function over $F$ is $\mathcal{N} \mathcal{P}$-hard. Next we provide a useful characterization of the optimal solutions to the problem of optimizing a linear function over the continuous relaxation of $F$. In Sect. 3 we discuss the classical submodular inequality formulation [21] of $F$ and derive two new classes of valid inequalities by lifting. We show that the lifting problems for the submodular inequalities are submodular maximization problems with special structure and are solvable by a greedy algorithm. This leads to an easily-computable strengthening by subadditive lifting of the submodular inequalities. Finally, in Sect. 5 we present computational results demonstrating the effectiveness of the new formulation with the proposed inequalities. 


\section{Preliminaries}

Because $f$ is differentiable and increasing, its inverse $g:=f^{-1}$ exists and is differentiable; and because $f$ is strictly concave increasing, $g$ is a strictly convex increasing function. It is convenient to rewrite $F$ as

$$
F=\left\{x \in\{0,1\}^{N}, w \in \mathbb{R}: a x \geq g(w)-d\right\} .
$$

We assume without loss of generality that $a>0$ since binary variables can be complemented. Note that complementing variables requires updating the constant term $d$ appropriately. Because $g(w)$ is an unrestricted real, the mixed 0-1 set $F$ may appear to be related to

$$
F^{\prime}:=\left\{x \in\{0,1\}^{N}, y \in \mathbb{R}: a x \geq y-d\right\}
$$

however, its structure is much richer than $F^{\prime}$. Observe that the continuous relaxation of $F^{\prime}$ has no fractional extreme points; thus, optimizing a linear function over $F^{\prime}$ is trivial. On the other hand, the convex function $g$ introduces infinitely many fractional extreme points to the continuous relaxation of $F$. Indeed, optimization of a linear function over $F$ is $\mathcal{N} \mathcal{P}$-hard. We elaborate on these points in the remainder of this section. We should remark that valid inequalities from $0-1$ knapsack $[3,11,27]$ or continuous 0-1 knapsack [18] sets are not useful for $F$ as $g(w)$ is unrestricted in sign. Let $\operatorname{conv}(F)$ denote the convex hull of $F$ and $\operatorname{relax}(F)$ denote the continuous relaxation of $F$ obtained by replacing the integrality restrictions on $x$ with constraints $\mathbf{0} \leq x \leq \mathbf{1}$. Throughout, for $S \subseteq N$ let $a(S):=\sum_{i \in S} a_{i}$.

\subsection{Optimization complexity}

Consider maximizing a linear function $w-c x$ over $F$ for the specific function $f(a)=$ $-e^{-a}$ (the exponential utility):

$$
\max \left\{w-c x: a x \geq g(w)=-\ln (-w), x \in\{0,1\}^{N}, w \in \mathbb{R}\right\}
$$

or, equivalently,

$$
\max \left\{-c x-e^{-a x}: x \in\{0,1\}^{N}\right\}
$$

Proposition 1 Optimization problem (3) is $\mathcal{N} \mathcal{P}$-hard.

Proof The proof is by reduction from PARTITION [9]. Given numbers $a_{i}, i \in N$, PARTITION calls for a subset $S$ of $N$ such that $a(S)=a(N \backslash S)$. If $a(N)=0$, the answer is trivially $S=\emptyset$. Otherwise, by scaling and negating if necessary, we may 
assume without loss of generality that $a(N)=-2$. Then, there exists $S$ such that $a(S)=a(N \backslash S)=-1$ if and only if the optimal value of the problem

$$
\max \left\{-e \cdot a x-e^{-a x}: x \in\{0,1\}^{N}\right\}
$$

equals 0 , which is true if and only if there is an optimal solution satisfying $a x=-1$.

\subsection{Continuous relaxation}

We now consider optimizing a linear function over the continuous relaxation of $F$ :

$$
\begin{gathered}
\max \{w-c x:(x, w) \in \operatorname{relax}(F)\}, \text { where } \\
\operatorname{relax}(F):=\left\{x \in \mathbb{R}^{N}, w \in \mathbb{R}: a x \geq g(w)-d, \mathbf{0} \leq x \leq \mathbf{1}\right\} .
\end{gathered}
$$

We employ (4) in the lifting and separation of inequalities in the subsequent sections. Because $g$ is a convex function, (4) is a convex optimization problem. Without loss of generality, we may assume that $c_{i} / a_{i}, i \in N$, are distinct, since otherwise two variables $x_{i}$ and $x_{j}$ with $c_{i} / a_{i}=c_{j} / a_{j}$ can be merged into a single variable $x_{k}$ with $c_{k}=c_{i}+c_{j}$ and $a_{k}=a_{i}+a_{j}$ without changing the problem.

Proposition 2 There is a unique optimal solution ( $x, w)$ to (4); moreover,

$$
x_{i}= \begin{cases}0, & \text { if } c_{i} / a_{i}>1 / g^{\prime}(w), \\ \frac{g(w)-a(T)-d}{a_{i}}, & \text { if } c_{i} / a_{i}=1 / g^{\prime}(w), \quad i \in N, \\ 1, & \text { if } c_{i} / a_{i}<1 / g^{\prime}(w),\end{cases}
$$

where $T=\left\{k \in N: x_{k}=1\right\}$.

Proof Because constraints of problem (4) are convex and relax $(F)$ has an interior point; e.g., $\left(\frac{1}{2} \mathbf{1}, g^{-1}\left(\frac{1}{2} a \mathbf{1}+d-1\right)\right)$, constraint qualification holds. Defining a dual variable $\lambda$ for $a x \geq g(w)-d, \alpha_{i}$ for $x_{i} \leq 1$, and $\beta_{i}$ for $-x_{i} \leq 0, i \in N$, KKT conditions imply

$$
\begin{gathered}
x_{i}: c_{i}=\lambda a_{i}-\alpha_{i}+\beta_{i} \\
w: 1=\lambda g^{\prime}(w) \\
\lambda, \alpha, \beta \geq \mathbf{0} .
\end{gathered}
$$

From complementary slackness we have that $x_{i}=1$ implies $\beta_{i}=0$ and $c_{i} / a_{i} \leq \lambda$, $x_{i}=0$ implies $\alpha_{i}=0$ and $c_{i} / a_{i} \geq \lambda$, whereas $0<x_{i}<1$, implies $\alpha_{i}=\beta_{i}=0$ and $\lambda=\frac{c_{i}}{a_{i}}=\frac{1}{g^{\prime}(w)}>0$. Because $g$ is strictly convex and the ratios $c_{i} / a_{i}$ are distinct, there is a unique solution satisfying these conditions.

Corollary 1 Each extreme point $(x, w)$ of relax $(F)$ has at most one fractional component $x_{i}, i \in N$. 
Fig. 1 A simple linearization cut (5)

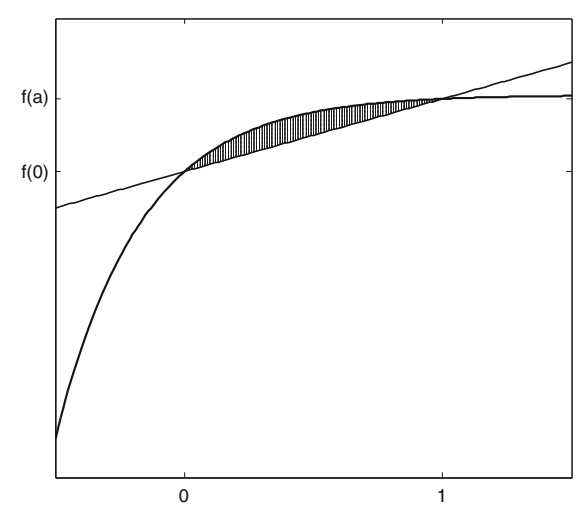

\section{Valid inequalities}

In this section we derive strong valid inequalities for $F$. In order to simplify the notation, in the remainder of the paper, we assume without loss of generality that $d=0$, since otherwise, we can work with $\tilde{g}(w)=g(w)-d$ and $\tilde{f}=\tilde{g}^{-1}$. Note that, like $g, \tilde{g}$ is differentiable, increasing, and strictly convex and, like $f, \tilde{f}$ is differentiable, increasing, and strictly concave.

In order to give first some intuition on the valid inequalities that cut off fractional solutions of relax $(F)$, we consider the simple case of $F$ with a single binary variable

$$
F_{1}=\{x \in\{0,1\}, w \in \mathbb{R}: f(a x) \geq w \Longleftrightarrow a x \geq g(w)\} .
$$

Evaluating $f(a x)$ for $x=0$ and $x=1$, one sees that the affine inequality

$$
w \leq f(0)+(f(a)-f(0)) x
$$

is valid for $F_{1}$ and eliminates the fractional solutions of relax $\left(F_{1}\right)$. Figure 1 illustrates inequality (5) and the cut-off fractional solutions for the case with $f(a x)=-e^{-a x}$.

\subsection{Submodular formulation}

Inequality (5) can be generalized to higher dimensions by using submodularity.

Definition 1 A set function $h: 2^{N} \rightarrow \mathbb{R}$ is submodular on $N$ if

$$
h(S)+h(T) \geq h(S \cup T)+h(S \cap T) \text { for all } S, T \subseteq N .
$$

A submodular function can be characterized by its difference function $\rho_{i}(S):=$ $h(S \cup i)-h(S)$ for $S \subseteq N$ and $i \in N \backslash S$. A set function $h$ is submodular if and only if its difference function is nonincreasing; that is, $\rho_{i}(S) \geq \rho_{i}(T)$ for all $S \subseteq T \subseteq N$ and all $i \in N \backslash T$ [21, p. 662]. The following properties of submodular functions will be useful. 
Proposition 3 [22] If $h$ is a submodular function on $N$, then

(1) $h(T) \leq h(S)-\sum_{i \in S \backslash T} \rho_{i}(N \backslash i)+\sum_{i \in T \backslash S} \rho_{i}(S)$ for all $S, T \subseteq N$;

(2) $h(T) \leq h(S)-\sum_{i \in S \backslash T} \rho_{i}(S \backslash i)+\sum_{i \in T \backslash S} \rho_{i}(\emptyset)$ for all $S, T \subseteq N$.

Consider now the set function $h: 2^{N} \rightarrow \mathbb{R}$ defined as

$$
h(S):=f(a(S)) \text { for } S \subseteq N .
$$

Proposition 4 The set function $h$ is submodular if $a \geq \mathbf{0}$ or $a \leq \mathbf{0}$.

Proof If $a \geq \mathbf{0}$, for $S \subseteq T$ and $i \notin T$, we have

$$
\rho_{i}(S)=f\left(a(S)+a_{i}\right)-f(a(S)) \geq f\left(a(T)+a_{i}\right)-f(a(T))=\rho_{i}(T),
$$

which holds from $a(T) \geq a(S), a_{i} \geq 0$, and concavity of $f$. Similarly if $a \leq \mathbf{0}$, for $S \subseteq T$ and $i \notin T$, we have

$$
-\rho_{i}(S)=f(a(S))-f\left(a(S)+a_{i}\right) \leq f(a(T))-f\left(a(T)+a_{i}\right)=-\rho_{i}(T),
$$

which holds from $a(T) \leq a(S), a_{i} \leq 0$, and concavity of $f$.

Proposition 3 immediately implies the following submodular inequalities

$$
\begin{gathered}
w \leq h(S)-\sum_{i \in S} \rho_{i}(N \backslash i)\left(1-x_{i}\right)+\sum_{i \in N \backslash S} \rho_{i}(S) x_{i} \text { for all } S \subseteq N \\
w \leq h(S)-\sum_{i \in S} \rho_{i}(S \backslash i)\left(1-x_{i}\right)+\sum_{i \in N \backslash S} \rho_{i}(\emptyset) x_{i} \text { for all } S \subseteq N
\end{gathered}
$$

for $F$ provided that $a \geq \mathbf{0}$ or $a \leq \mathbf{0}$. Either set of the inequalities (6) or (7) can be used to formulate a submodular maximization problem as a linear mixed 0-1 program [21, p. 710]. Because we assume without loss of generality that $a \geq \mathbf{0}$, we have a linear formulation of $F$ using the submodular inequalities

$$
F=\left\{x \in\{0,1\}^{N}, w \in \mathbb{R}:(6) \text { or }(7)\right\}
$$

Observe that inequality (5) is the special case of submodular inequalities (6) and (7) with $S=\emptyset$.

Although in the case of a single variable, the submodular inequalities are sufficient to give $\operatorname{conv}(F)$, for high dimensions our computational experience using the submodular inequalities (6) and (7) has shown them to be not very effective except for very small instances. These results are summarized in Sect. 5 for an expected utility maximization problem. In the next subsections we derive stronger inequalities that 
better exploit the concavity of the function $f$. In order to do so, by complementing variables $x_{i}, i \in S \subseteq N$, we first rewrite $F$ as

$$
F=\left\{x \in\{0,1\}^{N}, w \in \mathbb{R}: \sum_{i \in S}-a_{i}\left(1-x_{i}\right)+\sum_{i \in N \backslash S} a_{i} x_{i} \geq g(w)-a(S)\right\} .
$$

In the next subsections, we will derive two classes of inequalities by considering different restrictions of $F$. The first class is obtained by fixing $x_{i}=1$ for all $i \in S$ and then lifting inequalities from this restriction; whereas the second class is obtained by fixing $x_{i}=0$ for all $i \in N \backslash S$ and then lifting the corresponding inequalities.

\subsection{Inequalities from the restriction $F(\emptyset, S)$}

Consider the restriction of $F$ by setting $x_{i}=1$ for all $i \in S$ :

$$
F(\emptyset, S):=\left\{x \in\{0,1\}^{N \backslash S}, w \in \mathbb{R}: \sum_{i \in N \backslash S} a_{i} x_{i} \geq g(w)-a(S)\right\} .
$$

It follows from (6) that

$$
w \leq h(S)+\sum_{i \in N \backslash S} \rho_{i}(S) x_{i}
$$

is valid for $F(\emptyset, S)$. It is easily checked that (8) defines a facet of $\operatorname{conv}(F(\emptyset, S))$. Inequality (8) can be extended to a valid inequality for $F$ by lifting it with $x_{i}, i \in S$. Toward this end, we write the corresponding lifting function

$$
\begin{aligned}
\zeta(\delta):=\max & w-\sum_{i \in N \backslash S} \rho_{i}(S) x_{i}-h(S) \\
\text { s.t. } & \sum_{i \in N \backslash S} a_{i} x_{i} \geq g(w)-a(S)-\delta \\
& x \in\{0,1\}^{N \backslash S}, w \in \mathbb{R},
\end{aligned}
$$

for $\delta \in \mathbb{R}_{-}$. From Proposition 4, the lifting problem $(L: S)$ involves maximization of a submodular function, which is $\mathcal{N} \mathcal{P}$-hard in general. Nevertheless, as we show next, this particular submodular maximization problem can be solved efficiently by the greedy algorithm. We introduce some notation to describe the algorithm and $\zeta(\delta)$ explicitly. Let $N \backslash S=:\{1,2, \ldots, m\}$, be indexed so that $a_{1} \geq a_{2} \geq \cdots \geq a_{m}$ and let $A_{k}=\sum_{i=1}^{k} a_{i}$ for $k=1, \ldots, m$, with $A_{0}=0$.

Proposition 5 Algorithm Greedy $(L: S)$ solves problem $(L: S)$.

Proof Let $(x, w)$ be an optimal solution to $(L: S)$ and $T=\left\{i \in N \backslash S: x_{i}=1\right\}$. Suppose $T$ does not satisfy the greedy order; that is, $a_{i}>a_{j}$ for some $j \in T$ and 


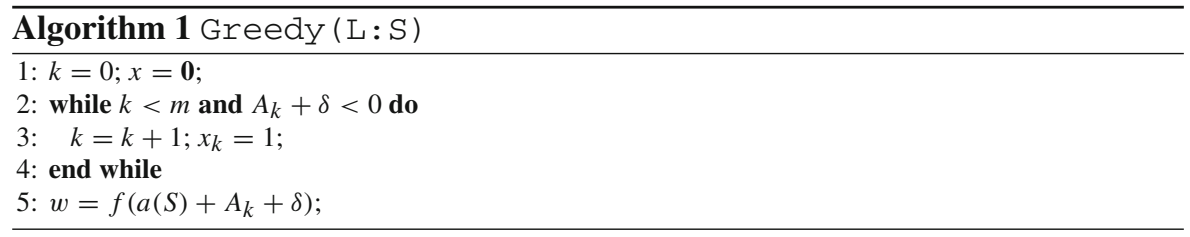

$i \notin T$. Then, consider the solution $T^{\prime}=T \cup i \backslash j$ and the corresponding objective values $z$ and $z^{\prime}$ for these solutions. We have

$$
\begin{aligned}
z^{\prime}-z= & f\left(a(T)+a(S)+\delta+a_{i}-a_{j}\right)-f(a(T)+a(S)+\delta)-\rho_{i}(S)+\rho_{j}(S) \\
= & f\left(a(T)+a(S)+\delta+a_{i}-a_{j}\right)-f(a(T)+a(S)+\delta) \\
& \quad-f\left(a(S)+a_{i}\right)+f\left(a(S)+a_{j}\right) \\
= & {\left[f\left(a(S)+a_{i}+a(T)-a_{j}+\delta\right)-f\left(a(S)+a_{i}\right)\right] } \\
& \quad-\left[f\left(a(S)+a_{j}+a(T)-a_{j}+\delta\right)-f\left(a(S)+a_{j}\right)\right]
\end{aligned}
$$

Then, because $a_{i}>a_{j}$ and $f$ is concave, $z^{\prime} \geq z$ if and only if $a(T \backslash j)+\delta \leq 0$.

Claim. Inequality $a(T \backslash i)+\delta \leq 0$ holds for all $i \in T$.

Suppose $\Delta:=a(T)-a_{i}+\delta>0$ for some $i \in T$. Consider the solution $T^{\prime \prime}=T \backslash i$ and its objective value $z^{\prime \prime}$. Then

$$
\begin{aligned}
z^{\prime \prime}-z & =f\left(a(T)+a(S)-a_{i}+\delta\right)-f(a(T)+a(S)+\delta)+\rho_{i}(S) \\
& =\left[f\left(a(S)+a_{i}\right)-f(a(S))\right]-\left[\left(f\left(a(S)+a_{i}+\Delta\right)-f(a(S)+\Delta)\right]>0,\right.
\end{aligned}
$$

where the inequality follows from $\Delta>0$ and strict concavity of $f$. However, this contradicts the optimality of $T$.

Claim. Either $a(T)+\delta \geq 0$ or $T=N \backslash S$.

Suppose $\Delta:=a(T)+\delta<0$ and $T \neq N \backslash S$; and let $i \in N \backslash(S \cup T)$. Consider the solution $\bar{T}=T \cup i$ and its objective value $\bar{z}$. Then

$$
\begin{aligned}
\bar{z}-z & =f\left(a(S)+a(T)+a_{i}+\delta\right)-\rho_{i}(S)-f(a(S)+a(T)+\delta) \\
& =\left[\left(f\left(a(S)+a_{i}+\Delta\right)-f(a(S)+\Delta)\right]-\left[f\left(a(S)+a_{i}\right)-f(a(S))\right]>0,\right.
\end{aligned}
$$

where the inequality follows from $\Delta<0$ and strict concavity of $f$. However, this contradicts the optimality of $T$.

It follows from Proposition 5 that the optimal value of the lifting problem $(L: S)$ can be stated as 
Fig. 2 Lifting function $\zeta$, its concave upper envelope $\gamma$, and the coefficients $\hat{\gamma}$ of inequality (6)

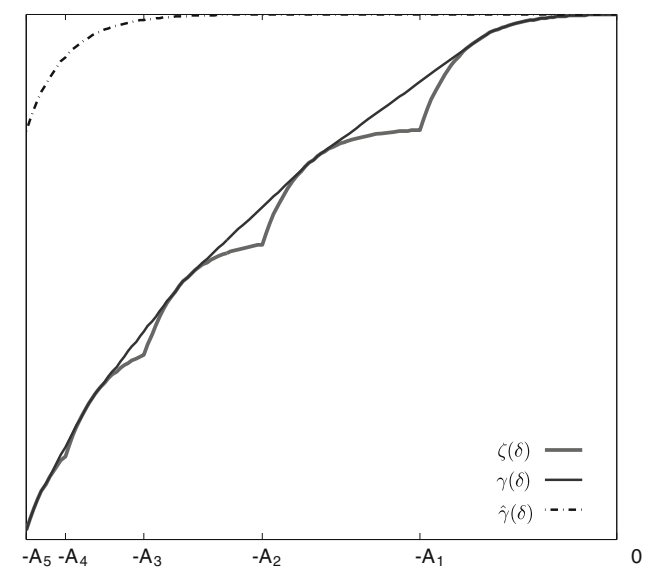

$$
\zeta(\delta)=f\left(a(S)+A_{k}+\delta\right)-\sum_{i=1}^{k} \rho_{i}(S)-f(a(S)),
$$

for $-A_{k} \leq \delta \leq-A_{k-1}, k=1, \ldots, m$.

The lifting function $\zeta$ is continuous on $\mathbb{R}_{-}$and concave on each interval [ $\left.-A_{k},-A_{k-1}\right], k=1, \ldots, m$ (see Fig. 2). However, it is not subadditive on $\mathbb{R}_{-}$. In order to construct a subadditive and, therefore, sequence-independent lifting function $[2,10,28]$, we consider the concave upper envelope of $\zeta$. Let

$$
\gamma(\delta):= \begin{cases}\zeta\left(\mu_{k}-A_{k-1}\right)-\rho_{k}(S) \frac{b_{k}(\delta)}{a_{k}}, & \text { if } \mu_{k}-A_{k} \leq \delta \leq \mu_{k}-A_{k-1}, \\ \zeta(\delta), & \text { otherwise, }\end{cases}
$$

where $\mu_{k}=g\left(\left(g^{\prime}\right)^{-1}\left(a_{k} / \rho_{k}(S)\right)\right)-a(S)$ and $b_{k}(\delta)=\mu_{k}-A_{k-1}-\delta$ for $k=1, \ldots, m$.

Proposition $6 \gamma$ is the concave upper envelope of $\zeta$ over $\mathbb{R}_{-}$.

Proof Let $\bar{\gamma}(\delta)$ be the upper bound of $\zeta(\delta)$ obtained by dropping the integrality restrictions in the lifting problem $(L: S)$. That $\bar{\gamma}$ is a concave upper bound of $\zeta$ follows from the fact that it is the continuous relaxation of the lifting function, which is a parametric convex optimization problem. Because ordering variables in nonincreasing $\rho_{i}(S) / a_{i}$ is the same as ordering them in nonincreasing $a_{i}$, from Proposition 2, we see that $x$ variables are increased in the same greedy order in the exact lifting problem as well as in its continuous relaxation. Furthermore, from Proposition 2, for a given $\delta$ if the optimal solution $(x, w)$ has an index $k \in N$ such that $0<x_{k}<1$, then $1 / g^{\prime}(w)=\rho_{k}(S) / a_{k}$, and otherwise $x$ is integral, in which case, the continuous relaxation is exact, i.e., $\bar{\gamma}(\delta)=\zeta(\delta)$. In the former case, because the value of $w$ is fixed as $\left(g^{\prime}\right)^{-1}\left(a_{k} / \rho_{i}(S)\right)$ as $\delta$ varies in the interval $\mu_{k}-A_{k} \leq \delta \leq \mu_{k}-A_{k-1}$, the function $\bar{\gamma}(\delta)$ changes linearly with slope $\rho_{k}(S) / a_{k}$. Because $\bar{\gamma}(\delta)>\zeta(\delta)$ for 
$\mu_{1}-A_{1} \leq \delta \leq 0$, we have

$$
\gamma(\delta)= \begin{cases}\zeta(\delta), & \text { if } \mu_{1}-A_{1} \leq \delta \leq 0, \\ \bar{\gamma}(\delta), & \text { otherwise. }\end{cases}
$$

As $\zeta$ and $\bar{\gamma}$ are concave over these intervals, we have that $\gamma$ is a concave upper bound on $\zeta$.

That $\gamma$ cannot be improved follows from $\gamma(\delta)=\lambda \zeta\left(\mu_{k}-A_{k-1}\right)+(1-\lambda) \zeta\left(\mu_{k}-\right.$ $A_{k}$ ) for $\delta=\lambda a_{k}+\mu_{k}-A_{k-1}$ and $0 \leq \lambda \leq 1$, i.e., $\gamma(\delta)$ is the convex combination of some $\zeta\left(\delta^{\prime}\right)$ and $\zeta\left(\delta^{\prime \prime}\right)$ whenever $\gamma(\delta)>\zeta(\delta)$.

Lemma 1 [12, p. 239] A concave function $\varphi: \mathbb{R}_{+} / \mathbb{R}_{-} \rightarrow \mathbb{R}$ is subadditive if and only if $\varphi(0) \geq 0$.

Because $\gamma \geq \zeta$ and from Lemma 1 it is subadditive on $\mathbb{R}_{-}$, we have

$$
\zeta\left(\sum_{i \in S}-a_{i} \bar{x}_{i}\right) \leq \gamma\left(\sum_{i \in S}-a_{i} \bar{x}_{i}\right) \leq \sum_{i \in S} \gamma\left(-a_{i}\right) \bar{x}_{i}
$$

implying the subadditive lifting inequality

$$
w \leq h(S)+\sum_{i \in S} \gamma\left(-a_{i}\right)\left(1-x_{i}\right)+\sum_{i \in N \backslash S} \rho_{i}(S) x_{i}
$$

is valid for $F$. A sufficient condition on the strength of the inequalities follows from the lifting argument.

Proposition 7 Inequality (9) is facet-defining for $\operatorname{conv}(F)$ if $\gamma\left(a_{i}\right)=\zeta\left(a_{i}\right)$ for all $i \in S$.

The next proposition shows that inequalities (9) are sufficient to cut off all fractional extreme points of the continuous relaxation of $F$.

Proposition 8 Inequalities (9) cut off all fractional extreme points of $\operatorname{relax}(F)$.

Proof Let $(x, w)$ be a fractional extreme point of relax $(F)$. From Proposition 2, $0<$ $x_{k}<1$ for some $k \in N, x_{i}=1$ for $T \subseteq N \backslash k$, and $x_{i}=0$ for $i \in N \backslash(T \cup k)$ and $g(w)=a(T)+a_{k} x_{k}$. Let us evaluate inequality (9) with $S=T$ for $(x, w)$ :

$$
\begin{aligned}
f\left(a(T)+a_{k} x_{k}\right) & \leq f(a(T))-\left(f\left(a(T)+a_{k}\right)-f(a(T))\right) x_{k} \\
& =\left(1-x_{k}\right) f(a(T))+x_{k} f\left(a(T)+a_{k}\right) .
\end{aligned}
$$

But this is not valid due to strict concavity of $f$.

Proposition 9 For each $S \subseteq N$ inequality (9) implies inequality (6). 
Proof For $a \leq 0$ letting $\hat{\gamma}(a):=f(a(N)+a)-f(a(N))$, we see that the coefficients $-\rho_{i}(N \backslash i)=\hat{\gamma}\left(-a_{i}\right), i \in N \backslash S$. Because $\hat{\gamma}(0)=\gamma(0)=0$, it is sufficient to show that $\gamma^{\prime}(a) \geq \hat{\gamma}^{\prime}(a)$ for $a \leq 0$ to establish $\gamma(a) \leq \hat{\gamma}(a)$ on $\mathbb{R}_{-}$.

For all $a \leq 0, \hat{\gamma}^{\prime}(a)=f^{\prime}(a(N)+a)$. On the other hand,

$$
\gamma^{\prime}(a)= \begin{cases}\rho_{k}(S) / a_{k} & \text { if } \mu_{k}-A_{k} \leq a \leq \mu_{k}-A_{k-1}, \\ f^{\prime}\left(a(S)+A_{k}+a\right) & \text { otherwise. }\end{cases}
$$

As $f$ is concave and $a(S)+A_{k} \leq a(N)$, we have $f^{\prime}\left(a(S)+A_{k}+a\right) \geq f^{\prime}(a(N)+a)$. On the other hand, for $\mu_{k}-A_{k} \leq a \leq \mu_{k}-A_{k-1}$, by concavity of $\hat{\gamma}$ and linearity of $\gamma$ on this interval, it suffices to observe that $\gamma^{\prime}\left(\mu_{k}-A_{k}\right) \geq \hat{\gamma}^{\prime}\left(\mu_{k}-A_{k}\right)$. To see this, recall that by definition of $\mu_{k}$, we have $g(w)=\mu_{k}+a(S)$ for $w=\left(g^{\prime}\right)^{-1}\left(a_{k} / \rho_{k}(S)\right)$. Then

$$
g^{\prime}(w)=\frac{a_{k}}{\rho_{k}(S)}=\frac{1}{f^{\prime}(g(w))} \quad\left(\text { as } g=f^{-1}\right)
$$

and $f^{\prime}\left(\mu_{k}+a(S)\right)=\rho_{k}(S) / a_{k}$. Then,

$$
\gamma^{\prime}\left(\mu_{k}-A_{k}\right)=f^{\prime}\left(\mu_{k}+a(S)\right) \geq f^{\prime}\left(a(N)-A_{k}+\mu_{k}\right)=\hat{\gamma}^{\prime}\left(\mu_{k}-A_{k}\right)
$$

by concavity of $f$.

The function $\hat{\gamma}$ is compared with the subadditive lifting function $\gamma$ in Fig. 2.

\subsection{Inequalities from the restriction $F(N \backslash S, \emptyset)$}

Consider the restriction of $F$, this time, by setting $x_{i}=0$ for all $i \in N \backslash S$ :

$$
F(N \backslash S, \emptyset)=\left\{x \in\{0,1\}^{S}, w \in \mathbb{R}: \sum_{i \in S}-a_{i}\left(1-x_{i}\right) \geq g(w)-a(S)\right\}
$$

It follows from (7) that

$$
w \leq h(S)-\sum_{i \in S} \rho_{i}(S \backslash i)\left(1-x_{i}\right)
$$

is valid for $F(N \backslash S, \emptyset)$. Furthermore, (10) is facet-defining for $\operatorname{conv}(F(N \backslash S, \emptyset))$. Inequality (10) can be extended to a valid inequality for $F$, this time by lifting it with $x_{i}, i \in N \backslash S$. To do so we compute the lifting function

$$
\begin{gathered}
\xi(\delta):=\max \quad w+\sum_{i \in S} \rho_{i}(S \backslash i)\left(1-x_{i}\right)-h(S) \\
(L: N \backslash S) \quad \text { s.t. } \sum_{i \in S}-a_{i}\left(1-x_{i}\right) \geq g(w)-a(S)-\delta \\
x \in\{0,1\}^{S}, w \in \mathbb{R}
\end{gathered}
$$


for $\delta \in \mathbb{R}_{+}$. Although the lifting problem $(L: N \backslash S)$ is a maximization of a submodular function, similar to problem $(L: S)$, it can also be solved efficiently by the greedy algorithm. In order to describe the algorithm and state $\xi(\delta)$ explicitly, let $S=:\{1,2, \ldots, r\}$, be indexed so that $a_{1} \geq a_{2} \geq \cdots \geq a_{r}$ and let $A_{k}=\sum_{i=1}^{k} a_{i}$ for $k=1, \ldots, r$, with $A_{0}=0$.

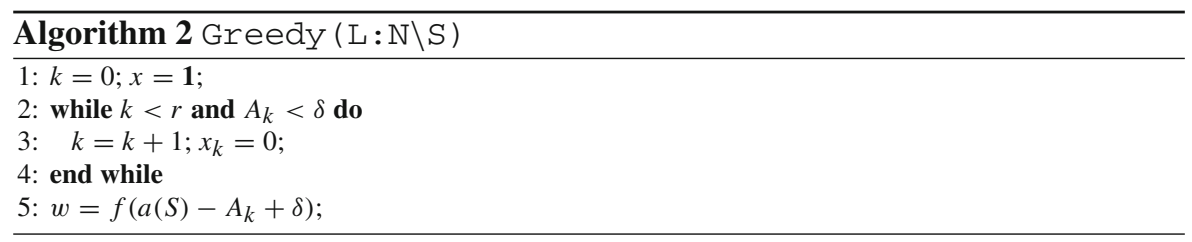

Proposition 10 Algorithm Greedy $(L: N \backslash S)$ solves problem $(L: N \backslash S)$.

Proof Let $(x, w)$ be an optimal solution to $(L: N \backslash S)$ and $T=\left\{i \in S: x_{i}=0\right\}$. Suppose $T$ does not satisfy the greedy order; that is, $a_{i}>a_{j}$ for $j \in T$ and $i \notin T$. Then, consider the solution $T^{\prime}=T \cup i \backslash j$ and the corresponding objective values $z$ and $z^{\prime}$ for these solutions. We have

$$
\begin{aligned}
z^{\prime}-z= & f\left(a(S \backslash T)-a_{i}+a_{j}+\delta\right)-f(a(S \backslash T)+\delta) \\
& +f(a(S))-f(a(S \backslash i))-f(a(S))+f(a(S \backslash j)) \\
= & {[f(a(S \backslash i)-a(T \backslash j)+\delta)-f(a(S \backslash j)-a(T \backslash j)+\delta)] } \\
& -[f(a(S \backslash i))-f(a(S \backslash j))]
\end{aligned}
$$

Then, because $a_{i}>a_{j}$ and $f$ is concave, $z^{\prime} \geq z$ if and only if $a(T \backslash j)-\delta \leq 0$.

Claim. Inequality $a(T \backslash i) \leq \delta$ holds for all $i \in T$.

Suppose $\Delta:=a(T \backslash i)-\delta>0$ for some $i \in T$. Consider the solution $T^{\prime \prime}=T \backslash i$ and its objective value $z^{\prime \prime}$. Then

$$
\begin{aligned}
z^{\prime \prime}-z & =f\left(a(S \backslash T)+a_{i}+\delta\right)-f(a(S \backslash T)+\delta)-(f(a(S))-f(a(S \backslash i))) \\
& =[f(a(S)-\Delta)-f(a(S \backslash i)-\Delta)]-[f(a(S))-f(a(S \backslash i))]>0,
\end{aligned}
$$

where the inequality follows from $\Delta>0$ and strict concavity of $f$, contradicting the optimality of $T$.

Claim. Either $a(T) \geq \delta$ or $T=S$.

Suppose $\Delta:=\delta-a(T)>0$ and $T \neq S$; and let $i \in S \backslash T$. Consider the solution $\bar{T}=T \cup i$ and its objective value $\bar{z}$. Then

$$
\begin{aligned}
\bar{z}-z & =f\left(a(S \backslash T)-a_{i}+\delta\right)+\rho_{i}(S \backslash i)-f(a(S \backslash T)+\delta) \\
& =[f(a(S))-f(a(S \backslash i))]-[f(a(S)+\Delta)-f(a(S \backslash i)+\Delta)]>0,
\end{aligned}
$$

where the inequality follows from $\Delta>0$ and strict concavity of $f$. However, this contradicts the optimality of $T$. 
By Proposition 10 the optimal value of the lifting problem $(L: N \backslash S)$ equals

$$
\xi(\delta)=f\left(a(S)-A_{k}+\delta\right)+\sum_{i=1}^{k} \rho_{i}(S \backslash i)-f(a(S)),
$$

for $A_{k-1} \leq \delta \leq A_{k}$ and $k=1, \ldots, r$.

The lifting function $\xi$ is continuous on $\mathbb{R}_{+}$and is concave on each interval $\left[A_{k-1}\right.$, $\left.A_{k}\right], k=1, \ldots, r$. However, it is not subadditive. In order to construct a subadditive lifting function, this time we consider the concave upper envelope of $\xi$. Let

$$
\omega(\delta):= \begin{cases}\xi\left(A_{k}-v_{k}\right)-\rho_{i}(S \backslash k) \frac{b_{k}(\delta)}{a_{k}}, & \text { if } A_{k-1}-v_{k} \leq \delta \leq A_{k}-v_{k}, \\ \xi(\delta), & \text { otherwise, }\end{cases}
$$

where $v_{k}=a(S)-g\left(\left(g^{\prime}\right)^{-1}\left(a_{k} / \rho_{k}(S \backslash k)\right)\right)$ and $b_{k}(\delta)=A_{k}-v_{k}-\delta$ for $k=1, \ldots, r$.

Proposition $11 \omega$ is the concave upper envelope of $\xi$ over $\mathbb{R}_{+}$.

The proof of Proposition 11 is similar to that of Proposition 6 and is omitted for brevity. As $\omega \geq \xi$ and it is subadditive on $\mathbb{R}_{+}$by Lemma 1 , we have

$$
\xi\left(\sum_{i \in N \backslash S} a_{i} x_{i}\right) \leq \omega\left(\sum_{i \in N \backslash S} a_{i} x_{i}\right) \leq \sum_{i \in N \backslash S} \omega\left(a_{i}\right) x_{i}
$$

and therefore the subadditive lifting inequality $[2,10,28]$

$$
w \leq h(S)-\sum_{i \in S} \rho_{i}(S \backslash i)\left(1-x_{i}\right)+\sum_{i \in N \backslash S} \omega\left(a_{i}\right) x_{i}
$$

is valid for $F$. From the lifting argument a sufficient facet condition follows.

Proposition 12 Inequality (11) is facet-defining for $\operatorname{conv}(F)$ if $\omega\left(a_{i}\right)=\xi\left(a_{i}\right)$ for all $i \in N \backslash S$.

Similar to (9), lifting inequalities (11) are sufficient to cut off all fractional extreme points of the continuous relaxation of $F$ as well.

Proposition 13 Inequalities (11) cut off all fractional extreme points of relax $(F)$.

Proposition 14 For each $S \subseteq N$ inequality (11) implies inequality (7).

The proofs of Propositions 13 and 14 are similar to that of Propositions 8 and 9 and are omitted for brevity. 


\section{Constraint generation}

In this section we describe how to generate the inequalities discussed in the previous section on-the-fly within a branch-and-bound algorithm. Given a point $(\bar{w}, \bar{x}) \in$ $\mathbb{R}_{-} \times \mathbb{R}_{+}^{N}$ if $\bar{x} \in\{0,1\}^{N}$, then separation is trivial: the corresponding inequality with $S=\left\{i \in N: \bar{x}_{i}=1\right\}$ is violated if and only if $\bar{w}>h(S)$. An exact separation for binary $\bar{x}$ ensures that $w \leq h(S)$ holds for all $S \subseteq N$ and, thus, the algorithm terminates with a correct solution.

On the other hand, if $\bar{x} \notin\{0,1\}^{N}$, we employ a heuristic scheme to cut off $\bar{x}$. In this case, in order to find violated cuts we follow a hierarchical approach, in which we first search for violated inequalities of the form

$$
w \leq h(S)+\sum_{i \in N \backslash S} \rho_{i}(S) x_{i}
$$

and

$$
w \leq h(S)-\sum_{i \in S} \rho_{i}(S \backslash i)\left(1-x_{i}\right)
$$

and then write the corresponding cuts (6) and (7), or (9) and (11) for the chosen subset $S$.

We first consider inequality (12). For a given $(\bar{w}, \bar{x})$, we are interested in finding a set $S \subseteq N$ that maximizes the violation of the inequality

$$
\bar{w} \leq h(S)-\sum_{i \in N \backslash S} \rho_{i}(S) \bar{x}_{i}
$$

To simplify the problem, we divide the constraint by $h(S)$ and approximate $\frac{\rho_{i}(S)}{h(S)}$ as $\frac{\rho_{i}(\emptyset)}{h(\emptyset)}$. If necessary by adding a constant to $f$, we may assume that $f(0)=h(\emptyset)>0$. Note that this approximation is exact for the exponential utility function because

$$
\frac{\rho_{i}(S)}{h(S)}=\frac{e^{-a(S \cup i)}-e^{-a(S)}}{e^{-a(S)}}=\frac{\left(e^{-a_{i}}-e^{0}\right)}{1}=\frac{\rho_{i}(\emptyset)}{h(\emptyset)} .
$$

Then, for finding a violated inequality we can write the following maximization problem

$$
\max _{S \subseteq N} \sum_{i \in N \backslash S} \frac{\rho_{i}(\emptyset)}{h(\emptyset)} \bar{x}_{i}+\bar{w} \frac{1}{h(S)} .
$$

Introducing $z \in\{0,1\}^{N}$ as the indicator of $S$, problem (14) is equivalent to

$$
\max _{z \in\{0,1\}^{N}} \sum_{i \in N} \frac{\rho_{i}(\emptyset)}{h(\emptyset)} \bar{x}_{i}\left(1-z_{i}\right)+\bar{w} \frac{1}{f(a z)} .
$$


Since $1 / f$ is convex and decreasing and $\bar{w}<0,(15)$ is a submodular maximization problem of the form (3).

For inequalities (13), given $(\bar{w}, \bar{x})$, we search for a set $S \subseteq N$ that maximizes the violation of the inequality

$$
\bar{w} \leq h(S)-\sum_{i \in S} \rho_{i}(S \backslash i)\left(1-\bar{x}_{i}\right)
$$

This time, by approximating $\frac{\rho_{i}(S \backslash i)}{h(S)}$ as $\frac{\rho_{i}(\emptyset)}{h(i)}$, which is also exact for the exponential utility function, we can write a similar submodular maximization problem of the form (3):

$$
\max _{z \in\{0,1\}^{N}} \sum_{i \in N} \frac{\rho_{i}(\emptyset)}{h(i)}\left(1-\bar{x}_{i}\right) z_{i}+\bar{w} \frac{1}{f(a z)} .
$$

In our implementation, we find heuristic solutions to the separation problems (15) and (17) by rounding up and rounding down their continuous relaxation solutions described in Proposition 2 in a greedy fashion.

\section{Computations}

In this section we describe our computational experiments with using the inequalities of Sect. 3 for solving an expected utility maximization problem in capital budgeting. As in Sect. 1 , for a set $N$ of investment options, let $a_{j}, j \in N$, be the capital requirements. Let $v_{i} \in \mathbb{R}^{N}$ be the value of investments at some future time under scenario $i$ with probability $\pi_{i}, i=1, \ldots, m$. Then, using the exponential utility function $1-\exp (z / \lambda)$ with risk tolerance $\lambda$, the expected utility maximization problem can be stated as

$$
\max \left\{\sum_{i=1}^{m} \pi_{i}\left(1-\exp \left(-\frac{v_{i} x}{\lambda}\right)\right): a x \leq 1, x \in\{0,1\}^{N}\right\}
$$

where, by scaling, we let the available budget for investments equal to 1 .

Introducing a continuous variable $w_{i} \in \mathbb{R}$ for each scenario $i$, we can rewrite the problem equivalently as

$$
1+\max \left\{\pi w: a x \leq 1, w_{i} \leq-\exp \left(-\frac{v_{i} x}{\lambda}\right), i=1, \ldots, m, x \in\{0,1\}^{N}\right\}
$$

so that each utility constraint defines a set of the form $F$. Thus the utility constraints can be reformulated into linear inequalities using the submodular inequalities (6), (7) or the lifted inequalities (9), (11). 


\subsection{Data generation}

The data set for the experiments are generated as follows. The capital requirements $\left(a_{i}\right.$ 's) are generated from a continuous uniform distribution in between 0 and 0.2. As is customary in the financial literature, in order to compute the future values, we use a factor model for investment returns. It is well-established that the nonnegative, skewed, and fatter-tailed lognormal distribution describes investment returns better than the normal distribution. Therefore, we draw Monte Carlo samples from the lognormal return distribution using a single factor model

$$
\ln r_{j}=\alpha_{j}+\beta_{j} \ln f+\epsilon_{j}, j \in N,
$$

where $f$ is the lognormal factor return (say, return of the overall market or industry), $\alpha_{j}$ is the active return, $\beta_{j} \ln f$ is the passive return component, and $\epsilon_{j}$ is the normal error with mean zero. For $j \in N$ we generate $\alpha_{j}$ from a continuous uniform distribution in between 0.05 and 0.10 and $\beta_{j}$ from a continuous uniform distribution in between 0 and 1. For scenario $i(i=1, \ldots, m)$ we draw $\ln f_{i}$ from $\operatorname{Normal}(0.05,0.0025)$ and $\epsilon_{i j}$ from Normal $(0,0.0025)$. Consequently, the value of investment $j \in N$ under scenario $i$ is

$$
v_{i j}=r_{i j} a_{j}
$$

with probability $\pi_{i}=1 / m$ for $i=1, \ldots, m$.

\subsection{Experiments}

In order to test the effectiveness of inequalities we generate five random instances as described above for varying number of variables $(n)$, scenarios $(m)$, and risk tolerances $(\lambda)$. All experiments are performed using the MIP solver of CPLEX Version 11.0 on a $3.12 \mathrm{GHz} \times 86$ Linux workstation with $1 \mathrm{~GB}$ main memory. The search tree size limit is set to $1 \mathrm{~GB}$. In the implementation, we approximate the exponential utility function using 2.72 for the irrational number $e$. By default, a solution is reported as optimal if the optimality gap is within $0.01 \%$.

In Table 1 we present a summary of our experiments with submodular inequalities and lifted inequalities. For varying number of variables $(n)$, scenarios $(m)$, and risk tolerances $(\lambda)$, we report the number of cuts added (cuts), the percentage integrality gap at the root node (rgap), percentage gap between the best known upper bound and lower bound at termination (egap), the number of branch-and-bound nodes explored (nodes), and the CPU time spent in seconds (time). Each row of the table represents the average of five instances.

An immediate observation in Table 1 is that the risk tolerance $(\lambda)$ is the most critical factor affecting the bounds, consequently the solution quality and overall performance. The higher the $\lambda$, the lesser is the investor's risk aversion. As risk aversion increases with smaller $\lambda$, the nonlinearity of the objective becomes more of an acute problem leading to weaker bounds. This observation is consistent for small as well as large instances. The number of the variables and scenarios does not appear to have a signifi- 
Table 1 Comparing submodular and lifted formulations

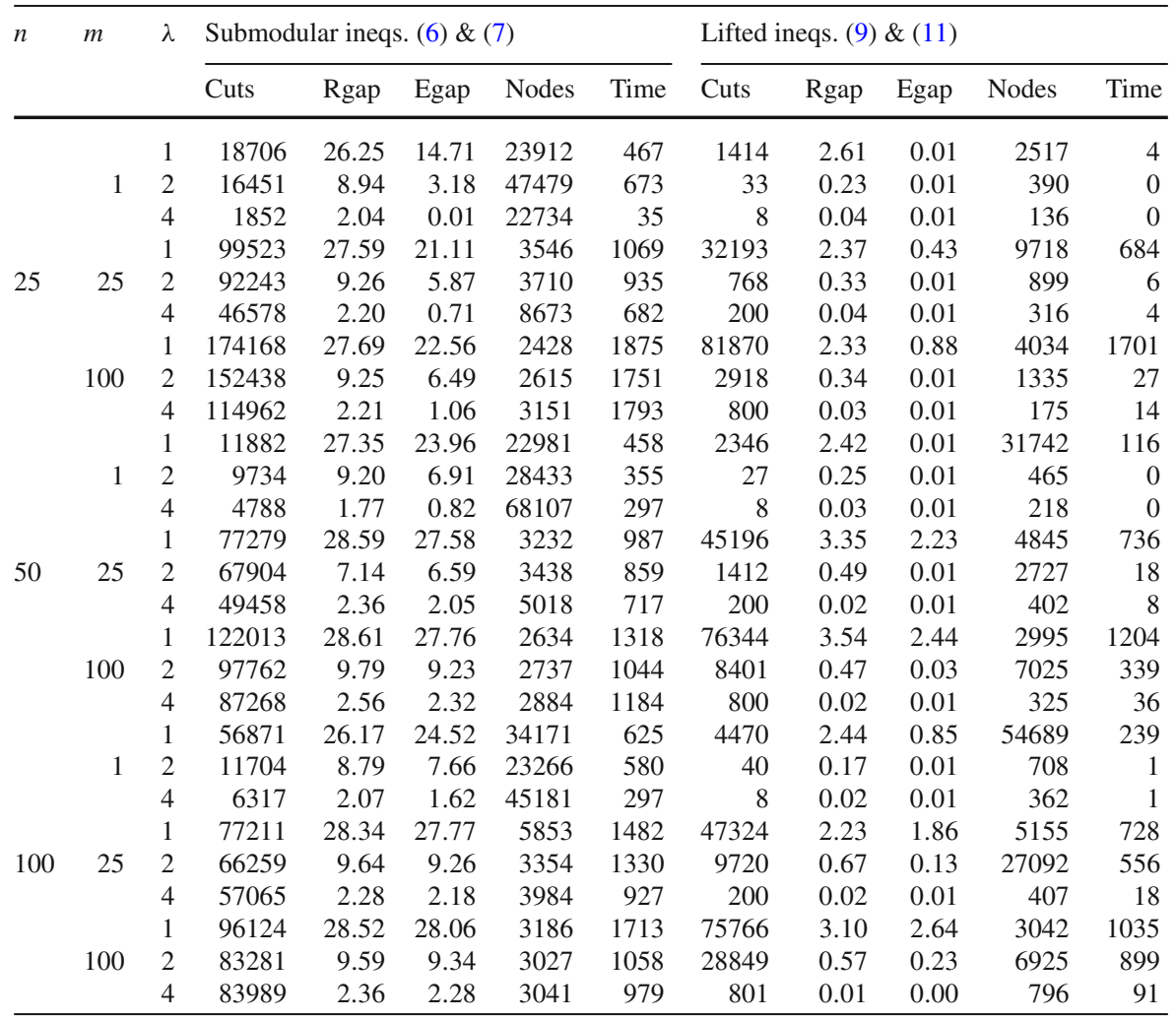

cant effect on the quality of the bounds. Additional experiments with controlling other parameters of the model ( $\alpha$ 's, $\beta$ 's, and variability) have shown that the effect of these parameters on the bounds and the performance of the algorithm is not as significant as the risk tolerance parameter $\lambda$.

Out of the 135 instances only 6 of the smallest instances are solved to optimality within the computational limits using the standard submodular formulation. All of the unsolved instances terminated due to memory limit of 1GB. For instances with 100 variables, the difference between the gaps at the root node and at termination is less than $2 \%$, indicating that no substantial progress was achieved to reduce the optimality gap even after extensive branching. The average root gap is $13.0 \%$ and the average optimality gap at termination is $10.9 \%$. From these results it is clear that the standard submodular formulation is not effective in tackling this problem except for very small instances or when the investor is close to being risk-neutral.

On the other hand, when formulated using the lifted inequalities the integrality gap at the root node and the search tree size are much smaller. Of the 135 instances 96 are solved to optimality. The average root gap is reduced from 13.0 to only $1.0 \%$ and the average optimality gap at termination is reduced from 10.9 to only $0.4 \%$. 
Table 2 Average of coefficients $(n=25, m=100, \lambda=1)$

\begin{tabular}{|c|c|c|c|c|c|c|c|}
\hline \multicolumn{4}{|c|}{ Inequalities (6) \& (9) } & \multicolumn{4}{|c|}{ Inequalities (7) \& (11) } \\
\hline$-\rho_{i}(N \backslash i)$ & $\gamma\left(-a_{i}\right)$ & $\zeta\left(-a_{i}\right)$ & $\operatorname{Imp}(\%)$ & $\rho_{i}(\emptyset)$ & $\omega\left(a_{i}\right)$ & $\xi\left(a_{i}\right)$ & $\operatorname{Imp}(\%)$ \\
\hline-0.112 & -0.288 & -0.292 & 98.88 & 1.478 & 0.398 & 0.396 & 99.78 \\
\hline
\end{tabular}

The comparison in Table 1 illustrates that the strengthening of the submodular inequalities through subadditive lifting is quite effective. In order to have a closer look at how much strengthening is achieved in the coefficients, in Table 2 we report the average of coefficients computed while running the algorithm for the instances with 25 variables, 100 scenarios and risk aversion $\lambda=1$. Recall that the coefficients $-\rho_{i}(N \backslash i)$ of the submodular inequality (6) are improved to $\gamma\left(-a_{i}\right)$ in the subadditive lifting inequality (9), whereas $\zeta\left(-a_{i}\right)$ is the lower bound given by the lifting function $\zeta$. In the table we see that $98.88 \%$ of the gap between $-\rho_{i}(N \backslash i)$ and $\zeta\left(-a_{i}\right)$ is closed by the subadditive lifting coefficients. Similarly, the coefficients $\rho_{i}(\emptyset)$ of the submodular inequality (7) are improved to $\omega\left(a_{i}\right)$ in the subadditive lifting inequality (11), whereas $\xi\left(a_{i}\right)$ is the lower bound given by the lifting function $\xi$. Here we observe that $99.78 \%$ of the gap between $\rho_{i}(\emptyset)$ and $\xi\left(a_{i}\right)$ is closed by the subadditive lifting coefficients. The magnitude of the difference between the coefficients of the submodular inequalities and the lifted inequalities is helpful in explaining the relative effectiveness of the inequalities observed in Table 1.

\section{Concluding remarks}

In this paper we studied a mixed-integer set that arises in combinatorial optimization problems with submodular utility maximization objectives, such as risk-averse capital budgeting under uncertainty, competitive facility location, and combinatorial auctions. The classical submodular reformulation of such problems into mixed 0-1 programming appears to be computationally ineffective due to its weak linear programming relaxation. In order to address this difficulty we strengthen the coefficients of the submodular inequalities by subadditive lifting that exploits the special structure of the particular submodular function. Computational experiments on expected utility maximization in capital budgeting show the effectiveness of the new formulation.

Acknowledgements The authors are thankful to the associate editor and a referee for their constructive comments.

Open Access This article is distributed under the terms of the Creative Commons Attribution Noncommercial License which permits any noncommercial use, distribution, and reproduction in any medium, provided the original author(s) and source are credited.

\section{References}

1. Aboolian, R., Berman, O., Krass, D.: Competitive facility location model with concave demand. Eur. J. Oper. Res. 181, 598-619 (2007) 
2. Atamtürk, A.: Sequence independent lifting for mixed-integer programming. Oper. Res. 52, 487-490 (2004)

3. Balas, E.: Facets of the knapsack polytope. Math. Program. 8, 146-164 (1975)

4. Berman, O., Krass, D.: Flow intercepting spatial interaction model: a new approach to optimal location of competitive facilities. Locat. Sci. 6, 41-65 (1998)

5. Corner, J.L., Corner, P.D.: Characteristics of decisions in decision analysis practice. J. Oper. Res. Soc. 46, 304-314 (1995)

6. Dobzinski, S., Nisan, N., Schapira, M.: Approximation algorithms for combinatorial auctions with complement-free bidders. In: STOC 2005 Proceedings of the 37th annual ACM symposium on theory of computing, pp. 610-618. ACM (2005)

7. Feige, U.: On maximizing welfare when utility functions are subadditive. In: STOC 2006 Proceedings of the thirty-eighth annual ACM symposium on theory of computing, pp. 41-50. ACM (2006)

8. Feige, U., Mirrokni V.S., Vondrák J.: Maximizing non-monotone submodular functions. In: FOCS 2007 Proceedings of the 48th annual IEEE symposium on foundations of computer science, pp. 461-471. $\operatorname{IEEE}(2007)$

9. Garey, M.R., Johnson, D.S.: Computers and Intractability: A Guide to the Theory of NP-Completeness. W. H. Freeman and Company, New York (1979)

10. Gu, Z., Nemhauser, G.L., Savelsbergh, M.W.P.: Sequence independent lifting in mixed integer programming. J. Comb. Optim. 4, 109-129 (2000)

11. Hammer, P.L., Johnson, E.L., Peled, U.N.: Facets of regular 0-1 polytopes. Math. Program. 8, 179206 (1975)

12. Hille, E., Phillips, R.S.: Functional Analysis and Semi-Groups. American Mathematical Society, Providence (1957)

13. Huff, D.L.: Defining and estimating a trade area. J. Mark. 28, 34-38 (1964)

14. Klastorin, T.D.: On a discrete nonlinear and nonseparable knapsack problem. Oper. Res. Lett. 9, 233237 (1990)

15. Lee, H., Nemhauser, G.L., Wang, Y.: Maximizing a submodular function by integer programming: polyhedral results for the quadratic case. Eur. J. Oper. Res. 94, 154-166 (1996)

16. Lehmann, B. Lehmann, D., Nisan, N.: Combinatorial auctions with decreasing marginal utilities. In: EC '01 Proceedings of the 3rd ACM conference on electronic Commerce, pp. 18-28. ACM (2001)

17. Lehmann, B., Lehmann, D., Nisan, N.: Combinatorial auctions with decreasing marginal utilities. Games Econ. Behav. 55, 270-296 (2006)

18. Marchand, H., Wolsey, L.A.: The 0-1 knapsack problem with a single continuous variable. Math. Program. 85, 15-33 (1999)

19. Mehrez, A., Sinuany-Stern, Z.: Resource allocation to interrelated risky projects using a multiattribute utility function. Manag. Sci. 29, 439-490 (1983)

20. Nemhauser, G.L., Wolsey, L.A.: Best algorithms for approximating the maximum of a submodular function. Math. Oper. Res. 3, 177-188 (1978)

21. Nemhauser, G.L., Wolsey, L.A.: Integer and Combinatorial Optimization. Wiley, New York (1988)

22. Nemhauser, G.L., Wolsey, L.A., Fisher, M.L.: An analysis of approximations for maximizing submodular set functions-I. Math. Program. 14, 265-294 (1978)

23. Schoemaker, P.J.H.: The expected utility model: its variants, purposes, evidence and limitations. J. Econ. Lit. 20, 529-563 (1982)

24. von Neumann, J., Morgenstern, O.: Theory of Games and Economic Behavior. Princeton University Press, Princeton (1947)

25. Vondrak, J.: Optimal approximation for the submodular welfare problem in the value oracle model. In: STOC 2008 Proceedings of the fourth annual ACM symposium on theory of computing, pp. 67-74. ACM (2008)

26. Weingartner, H.M.: Capital budgeting of interrelated projects: survey and synthesis. Manag. Sci. 12, 485-516 (1966)

27. Wolsey, L.A.: Faces for linear inequality in 0-1 variables. Math. Program. 8, 165-178 (1975)

28. Wolsey, L.A.: Valid inequalities and superadditivity for $0 / 1$ integer programs. Math. Oper. Res. 2, 6677 (1977)

29. Wolsey, L.A.: Submodularity and valid inequalities in capacitated fixed charge networks. Oper. Res. Lett. 8, 119-124 (1988) 